\title{
X/Ka-Band Dual-Polarized Digital Beamforming Synthetic Aperture Radar
}

\author{
Chunxu Mao, Steven Gao, Carolina Tienda, Srdjan Glisic, Emilio Arnieri, Piotr Penkala, Milos Krstic, \\ Luigi Boccia, Anton Patyuchenko, Arancha Dominuez, Fan Qin, Oliver Schrape, Marwan Younis, \\ Elisabeth Celton, Arkadiusz Koczor, Giandomenico Amendola, Tobias Rommel, Vladimir Petrovic, \\ Uroschanit Yodprasit
}

\begin{abstract}
This paper presents a novel digital beamforming (DBF) space-borne synthetic aperture radar (SAR) for future space-borne earth observation. The objective of the DBF-SAR system is to realize a next-generation space-borne SAR system for Europe, which has low cost, light weight, low power consumption, dual-band (X/Ka) dual-polarized operation and a compact size compatible with future small/micro satellites platforms. The concept and designs of the DBF multi-static SAR system are discussed first, followed by the designs of sub-systems such as digital beamforming networks (DBFN), MMIC and antennas are presented. Then some simulated and measured results of each sub-system are shown. The proposed SAR system has low cost and compact size and is promising for future SAR applications.
\end{abstract}

Index Terms - Dual-band, dual-polarized, digital beamforming (DBF), synthetic aperture radar (SAR).

\section{INTRODUCTION}

Space-borne (synthetic aperture radar) SAR is a multipurpose sensor that can be operated in earth observation (EO) in any weather conditions and all day/night. Traditionally, the SAR system in space is a mono-static system, which uses the same antenna for transmitting and receiving. Most of space-borne SAR systems are based on large-satellite platforms and make use of phase-arrays or mechanical steering, thus they suffer from the problems of

This paper is an expanded version from the 2015 Asia-Pacific Microwave Conference, Nanjing, China, Dec. 6-9, 2015. Manuscript submitted on Jan 31, 2016; This work is supported by the project "DIFFERENT" funded by EC FP7 (grant no. 6069923).

C. Mao, S. Gao and F. Qin are with School of Engineering and Digital Arts, University of Kent, Canterbury, UK (email: cm688@kent.ac.uk; s. gao@kent.ac.uk).

C. Tienda, A. Patyuchenko, M. Younis and T. Rommel are with Microwaves and Radar Institute, German Aerospace Center (DLR), 82234 Wessling, Germany.

S. Glisic, U. Yodprasit are with Silicon Radar GmbH, 15236 Frankfurt (Oder), Germany.

E. Arnieri, L. Boccia and G. Amendola are with DIMES, Universitàdella Calabria, 87036 Arcavacada di Rende Cosenza, Italy.

P. Penkala, A. Koczor are with Evatronix S.A. Bielsko-Biała, 43-300 Bielsko-Biała, Poland.

M. Krstic, O. Schrape and V. Petrovic are with IHP, 15236 Frankfurt (Oder), Germany.

A. Dominuez, E. Celton is with Innovative Solutions In Space BV, 629 JD, Delft, Netherlands. high cost, high power consumption and limited performance [1]. This paper will present a novel X/Ka-band digital beamforming SAR (DBF-SAR) system proposed in the project DIFFERENT. DIFFERENT is abbreviated of "digital beam forming for low-cost multi-static space-borne synthetic aperture radars". The project currently still in progress, is collaborated amongst several leading universities, research institutes and companies in Europe. The aim of DIFFERENT project is to develop a low-cost, low weight, highly integrated, dual-band dual polarizations DBF-SAR instrument to overcome the limitations of current SAR systems and pave the way to small satellites formation flying missions.

To solve the problems of traditional SAR systems, a multistatic SAR system based on formation flying small satellites is proposed in this paper. In this SAR system, the transmitting and receiving antennas are separated and mounted on separate satellites, enabling a lager freedom of operation and increasing the sensitivity due to the reduction of transmitter/receiver switches. This distributed multi-static SAR system will strongly support the use of small, low-cost satellites in the future [2]-[5]. The reduction of power demands of passive receivers will also enable an accommodation of radar payload on micro-satellites.

The DBF technique applied in SAR system is to reduce the cost, weight and power consumption in micro-satellites. In this concept, the receiving antenna is split into multiple subaperture and the received signals from each sub-aperture element are separately amplified, down-converted and digitized. Compared with analogue beam forming, DBF is much more powerful as it can form multiple steerable beams towards different targets simultaneously and adaptive beam shaping [6]. The DBF-SAR system can improve the radar performances with better sensitivity, lower ambiguity level and higher resolution over a wide swath. In addition, due to the multiple independent data channels, the operation flexibility can be enhanced. It is evaluated that DBF will be employed by next-generation of space-borne SAR missions such as Tandem-L [7], Sentinel-1 follow-on [8], NASA-ISRO [9] and HRWS [10]. An example of a potential Earth observation mission based on the SAR system in DIFFERENT has been illustrated in [11] [12].

Up to now, all of the SAR systems for small satellites are operating at single band, which limits SAR applications in 
more advanced EO mission. A shared-aperture, dual-band dual-polarized SAR radiation board will not only lead to a compact size, low cost SAR system, but also versatile applications. To meet the requirements of the future SAR missions, the bandwidth of each band should be larger than $5 \%$. Besides, the dual-band antenna with excellent cross polarization discrimination (XPD) and high isolation between elements are required.

This paper is organized as follows. Section II presents the state-of-the-art space-borne SAR systems and the DBF-SAR system in the project DIFFERENT. Section III presents the design of DBFN. Section IV presents the designs and results of MMIC and silicon manufacturing technologies. Section V presents the designs and results of the integrated feed using an $\mathrm{X} /$ Ka-band dual-polarized array and the whole antenna system followed by conclusion in Section VI.

\section{DBF-SAR SYSTEM}

\section{A. State-Of-The-Art Space-Borne SAR Systems}

There has been a considerable increase of EO applications that requires high-resolution SAR images. New SAR instruments must fulfill challenging requirements and enable the capability of acquiring images with both wide-swath and high resolution. The two key technologies considered to improve future SAR performance are digital beamforming and multi-aperture signal recording. An example of this approach is referred to as HRWS (high-resolution wide-swath) SAR which can cover $70 \mathrm{~km}$ swath with $1 \mathrm{~m}$ resolution [13].

In SAR applications, the radar pulse travel time and its arrival angle to the ground is directly associated. For every instant of time, the antenna gain in receiver can be optimized using real time beamforming in the direction the expected echo from ground is arriving. Digital beamforming on receiver denotes as SCORE (scan-on-received) process, which steers the narrow elevation beam on receiver in the desired direction. Large received antennas are frequently used to increase the sensitivity without reducing the swath width [14] [15].

To further improve the azimuth resolution than the conventional stripmap SAR, the receiver antenna can be divided into multiple sub-antennas along the track direction. Each antenna acquires several azimuth samples of echo from the transmitted pulse and sees a wider Doppler spectrum. Each aperture is connected to a received channel; the received signals are recorded and retransmitted to the ground for further post-processing [16]. A coherent combination of the signals from the different sub-apertures provides a unique high resolution SAR image. This technique has one limitation that fixed PRF (Pulse Repetition Frequency) is required. Between two consecutive transmit pulses, the satellite should move half distance of the length of the antenna [16]. This limitation can be overcome using multichannel data processing [18]-[20].

The HRWS SAR requires a large antenna apertures to cover a large swath areas. For every $100 \mathrm{~km}$ swath width, approximately $10 \mathrm{~m}$ aperture is required. To avoid the increase of the antenna size, new instruments have been developed [21]. In ScanSAR technique, different azimuth bursts are used to cover several swathes. The resolution loss of this approach

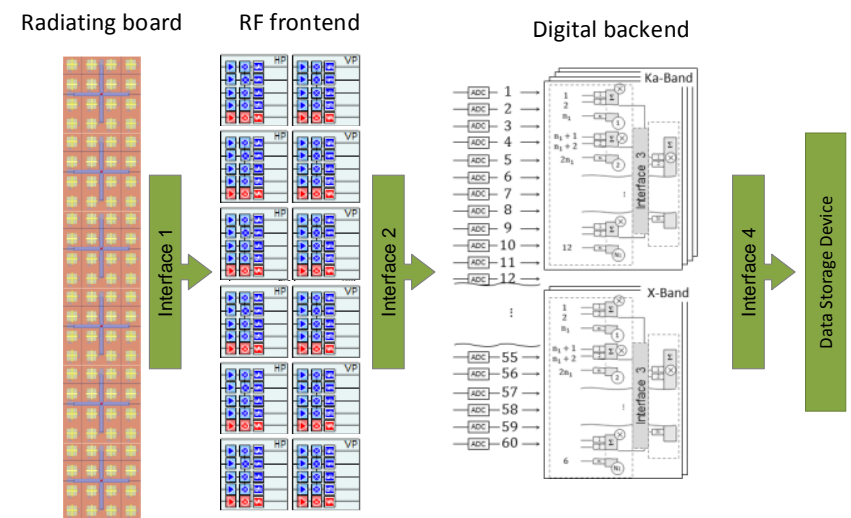

Fig. 1. Architecture of radar module in DIFFERENT.

is compensated using a wider Doppler spectrum. This system is considered by ESA to cover $400 \mathrm{~km}$ swath width with $5 \mathrm{~m}$ resolution in a project that will replace Sentinel-1 [22]. A drawback of the multichannel ScanSAR is that high Doppler centroid is required to meet the astringent resolution requirements.

Apart from multichannel ScanSAR, other alternative concepts have been considered to save the echoes arriving from different directions simultaneously. This concept increases the swath width without increasing the antenna size and bursts. Another interesting alternative are parabolic reflectors fed with a phased array. The reflector focuses the arriving echo and transmit it to the different channels of the feed [23] [24]. The feed elements are digitally combined, contributing to a multiple-beam technique. The main drawback of this mode is the blind ranges which is produced because the radar cannot transmit and receive simultaneously. This limitation can be overcome using a bi-static SAR where the pulse is transmitted with one satellite and received by the other [25]. Another alternative is to use a variation of PRF to shifts the blind ranges across the swath; however, additional data processing is required in this case [26].

\section{B. SAR System in DIFFERENT Project and Its Design}

The innovative SAR concept developed in DIFFERENT is based on digital beamforming (DBF) concept. DIFFERENT enables the realization of multiple advanced operational modes and make it innovative compared with current platforms. DIFFERENT has a dual-band (X- and Ka-bands) performance which enables it apply in new mission scenarios [29]. The project is planned for operating in a constellation with two or more satellites involved. The DIFFERENT concept mission could not only fly in tandem with an existing $\mathrm{X}$-band master satellite but also as a swarm of small platforms to collect the Ka-band data. The Ka-band sub-system of DIFFERENT can be extended into a compact single-pass interferometric system based on the same satellite platform. Due to the high Ka-band frequencies, it is possible to be realized within a single satellite spacecraft.

The architecture of DIFFERENT radar module demonstrator consists of four main blocks: RF board, analog to digital converters (ADC) board and digital board (DGT). These blocks are connected through interfaces, as shown in Fig. 1. The radiating board is composed of $6 \mathrm{X}$-band and 96 Ka-band (24 in elevation and 4 in azimuth) dual-polarized 


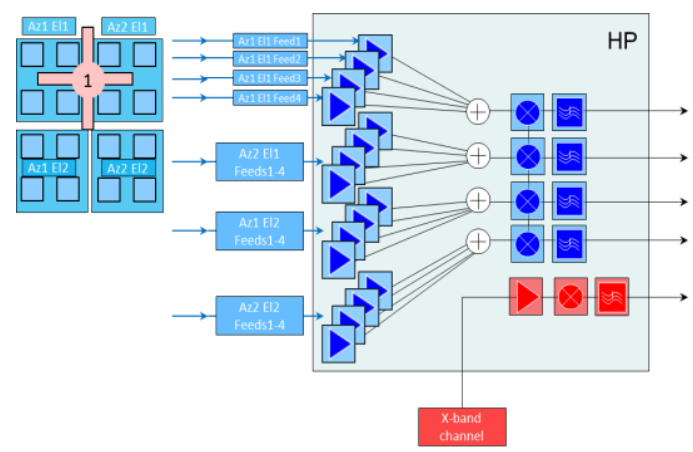

Fig. 2. Block diagram of active summation of the RF frontend.

antenna elements. Every $2 \times 2$ Ka-band elements are active combined and form a channel, as shown in Fig. 2. The function of each RF MMIC unit is to down-convert the received $\mathrm{V}$ - and $\mathrm{H}-$ pol signals to an intermediate frequency (IF) band. The down-converted signals are processed in the digital backend block, which contains 60 ADCs. After digitization, the IF signals are pre-processed in the Digital Beamforming Network (DBFN). There are in total $4 \times 2$ DBFN blocks integrated into 6 DGT boards. In each DBFN unit, the digitized data corresponding to all elevation channels, specific azimuth channel and polarization are weighted and combined.

The maximum power level for the X- and Ka-band subsystems is estimated using the radar equation for distributed targets,

$P_{r}=\frac{P_{t} G_{T} G_{R} \lambda^{2} \sigma_{0} \tau_{p} c}{2(4 \pi)^{3} L_{f}} \cdot \frac{\int_{\Phi}\left|C_{T}(\phi)\right|^{2}\left|C_{R}(\phi)\right|^{2} d \phi}{r^{3}(\vartheta) \sin \left(\eta_{i}\right)} \cdot \frac{1}{N}$

where $P_{t}$ is the transmit power, $G_{T}$ is the gain of the transmit antenna, $G_{R}$ is the gain of the receive antenna, $\lambda$ is the wavelength, $\sigma_{0}$ is the backscattering coefficient, $\tau_{p}$ is the pulse length, $\mathrm{c}$ is the speed of light, $\mathrm{L}_{\mathrm{f}}$ is the losses component, $\mathrm{C}_{\mathrm{T}}(\phi), \mathrm{C}_{\mathrm{R}}(\phi)$ are transmit and receive antenna patterns, $\phi$ is the antenna pattern angle, $r$ is the slant range, $\eta_{i}$ is the incidence angle of the signal, $\mathrm{N}$ is the number of reflector channels receiving the most of the power from the given direction. Using the (1), the maximum received power level by a single reflector channel can be estimated. To ensure a certain margin in the maximum power level, $\mathrm{N}=1$ is chosen. Thus, the results for both bands sub-systems of DIFFERENT are obtained. For X-band, the maximum and minimum receive power are $-62.9 \mathrm{dBm}$ and $-90.66 \mathrm{dBm}$ respectively and for Kaband, the results are $-70.85 \mathrm{dBm}$ and $-90.9 \mathrm{dBm}$, respectively.

The minimum power levels are defined as the noise level, which can be evaluated according to the following expression,

$$
P_{n}=k T B_{w}
$$

where $k$ is Boltzmann constant, $T$ is the noise temperature, $B_{w}$ is the signal bandwidth. The noise level depends on the final hardware of the module. Therefore the minimum power levels given in this section must be considered.

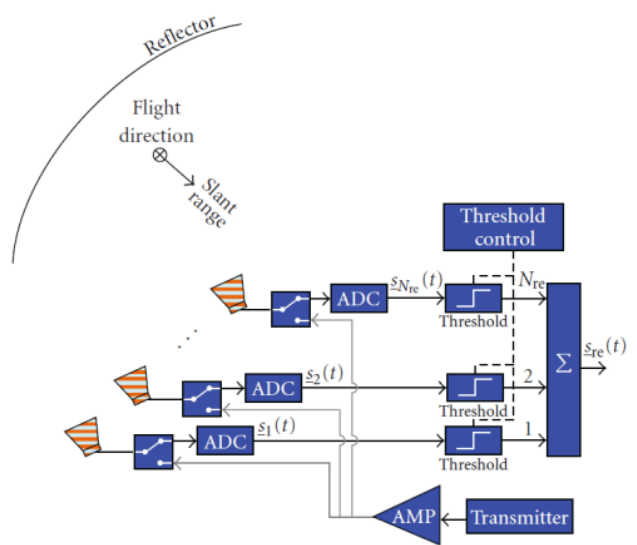

Fig. 3. System architecture of the reflector based DBF-SAR.

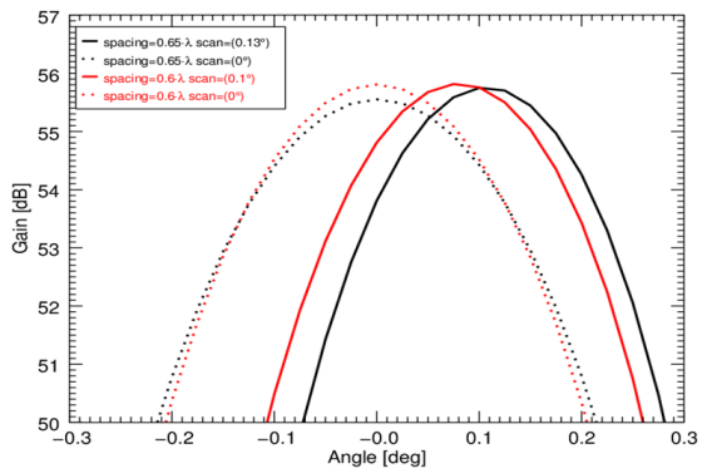

Fig. 4. Radiation patterns of different spacing between elements at $0^{\circ}$ (dashed line) and $0.13^{\circ}$ (solid line) with DBF post-processing used.

The reflector system is adopted in the DBF-SAR system, which consists of a parabolic reflector and a feed array of receive elements, as shown in Fig. 3. To illuminate a given angular segment in elevation, the corresponding feed elements are activated. In this case, DBF consists of selecting a subset of the feed elements and summing up the corresponding data streams weighted with complex coefficients $w_{i}(t)$. In a general case, the output signal in this case is represented by,

$$
s_{r e}(t)=\sum_{i=1}^{N_{r e}} w_{i}(t) \cdot s_{i}(t)
$$

$s_{i}(t)$ is the data stream of the channel $i, w_{i}(t)$ is the time varying complex coefficient, $s_{r e}(t)$ is the summed up output signal.

In the basic case, the complex weighting coefficients are equal to 0 (for non-activated feed elements) or 1 (for the activated feeds). Thus, the output signal is given by

$$
s_{r e}(t)=\sum_{i=n}^{n+N_{a c t}} s_{i}(t), n \geq 1, n+N_{a c t} \leq N_{r e}
$$

$N_{\text {act }}$ is the given number of adjacent active elements. The digital threshold detectors is used to determine whether a data stream is passed to the summation or nulled. For the SAR processing it is important to record the summed signal at each instance in order to reconstruct the actual antenna pattern.

Fig. 4 shows the simulated radiation patterns of the illuminated parabolic reflector with the DBF post-processing 


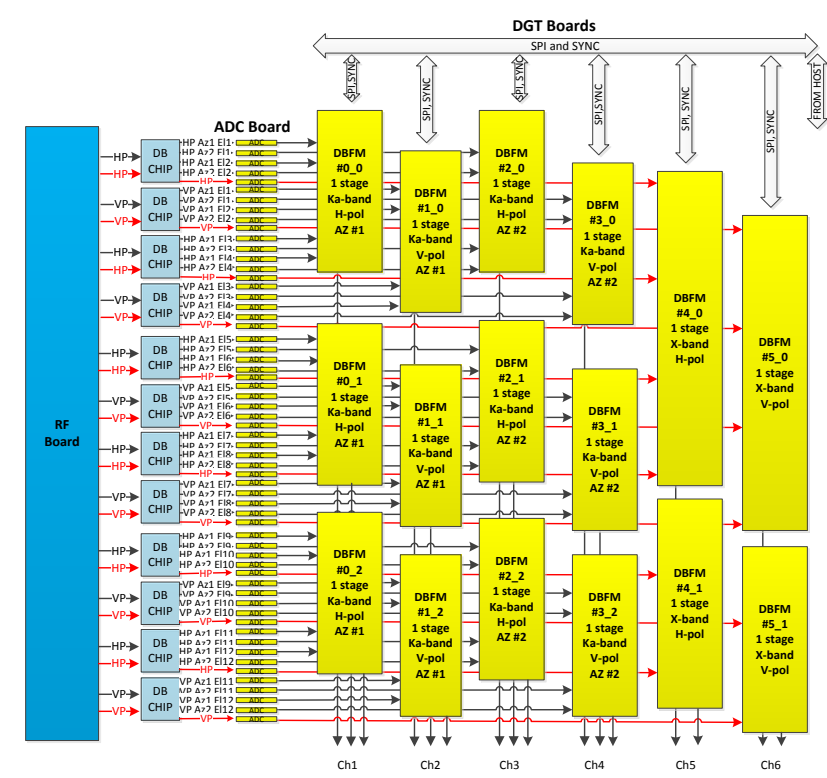

Fig. 5. System-level architecture of DBFN.

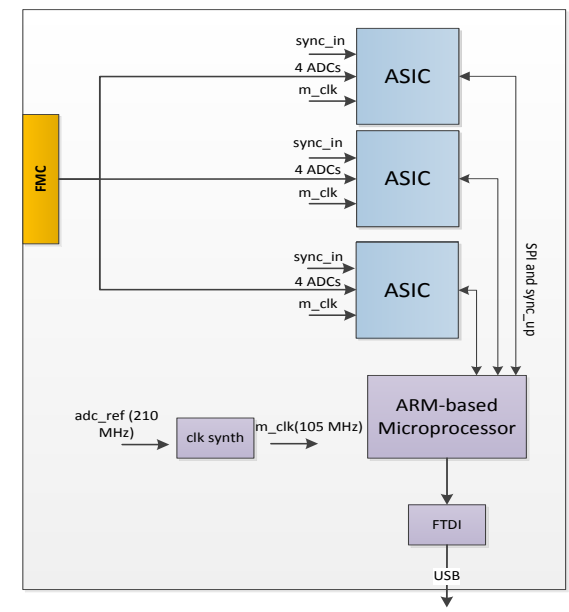

Fig. 6. Block diagram of the DGT board.

is applied. Two spacing of $0.65 \cdot \lambda_{K a}$ and $0.6 \cdot \lambda_{K a}$ between two consecutive elements are investigated. It is observed that the patterns at different scan angles and spacing exhibit a similar illumination performance (gain and HPBW) due to the complex DBF weights are employed.

\section{DIGITAL BEAMFORMING NETWORK}

The system architecture of the digital part of radar demonstrator is presented in Fig. 5. The DBFN is composed of the front-end and back-end network blocks. Each front-end module is connected to four ADCs, synchronization bus, one back-end chip and SPI bus. SPI serves the purpose of a configuration and LUT programing interface. The control unit manages the start/stop function and the changes of complex weight synchronization. The DBFN is a cluster of individual working DBF cores. The nodes are synchronized with each other using a synchronization interface. The length and type of acquisition process is configurable by the SPI interface. The system which covers 60 ADC converters requires 16 cores. The core can work in two modes: static mode and dynamic

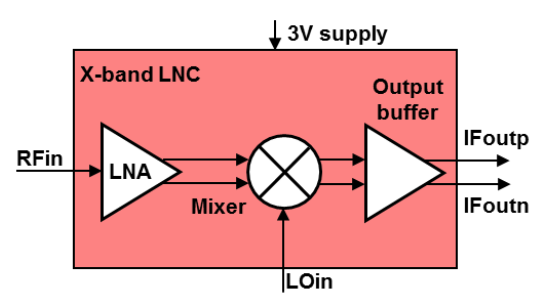

(a)

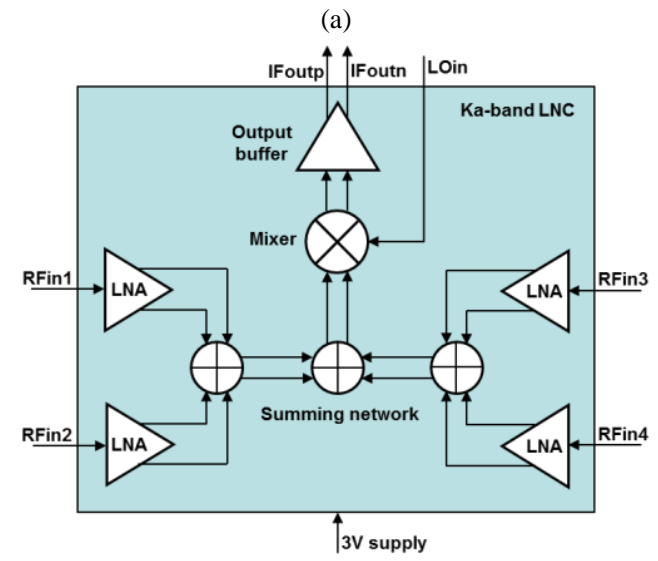

(b)

Fig. 7. Block diagram of the LNC: (a) X-band, (b) Ka-band.

mode. For static mode, the weights are fixed during operation whereas the weights can be changed in the dynamic mode. Then microprocessor adds up sub-streams to form an output stream for a given azimuth.

Fig. 6 shows the block diagram of DGT board. The digital backend is composed of an ARM-based micro-processor, three ASICs and several modules of clock synthesizer, FTDI module (FIFO to USB), SMA connector and FMC connectors. The design has been verified using Verilog test bench, which is based on model-based design using MATLAB. Depending on the test scenario, one or more periods of input signals are provided to the ADC interfaces. The results of physical implementation are listed as follow:

- Die Size: $6846.40 \times 6846.40 \mu \mathrm{m}$

- Num. of Instances: 625738

- Number of Flip-Flops: 14901

- TMR Flip-Flops: 4408

- Chip Area: $47 \mathrm{~mm}^{2}$

- SRAM Area: $15.08 \mathrm{~mm}^{2}$

- Power consumption < 1.67 Watt

The dynamic power consumption is based on stimuli of RTL simulation. It represents an average of over 10 cycles of the main active timing window of the laid out design.

\section{MMIC AND SILICON TECHNOLOGY}

The purpose of the analog monolithic microwave integrated circuit (MMIC) chips is to amplify and down-convert the received $\mathrm{X}$ - and Ka-band signals to IF band. The architecture of the X-and Ka-band low-noise converter (LNC) chips is shown in Fig. 7. The X-band LNC features a low noise amplifier (LNA), a mixer drove with an off-chip $9.6 \mathrm{GHz} \mathrm{LO}$ signal and an output buffer. Fig. 7(b) shows the block diagram of the Ka-band LNC. Each Ka-band LNC chip is connected to 


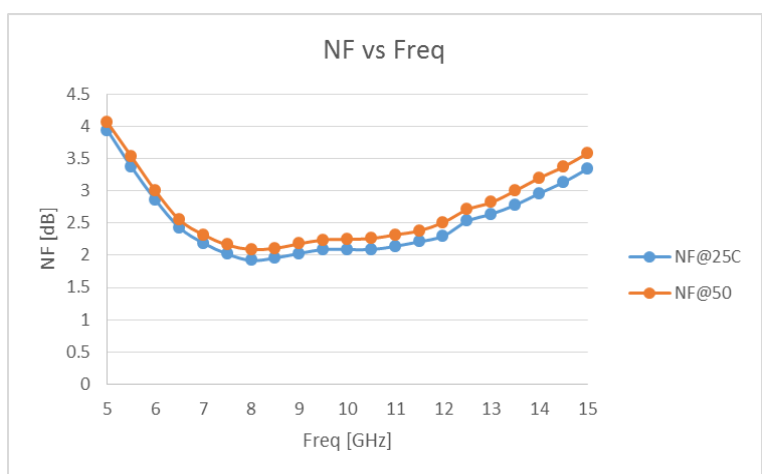

Fig. 8. Measured X-band LNA noise figure at 25 and $50{ }^{\circ} \mathrm{C}$.

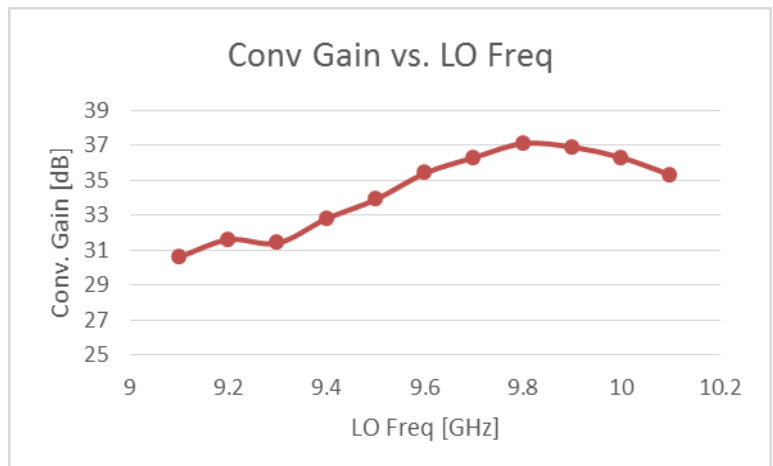

Fig. 9. Measured conversion gain of the X-band LNC vs. LO frequency.

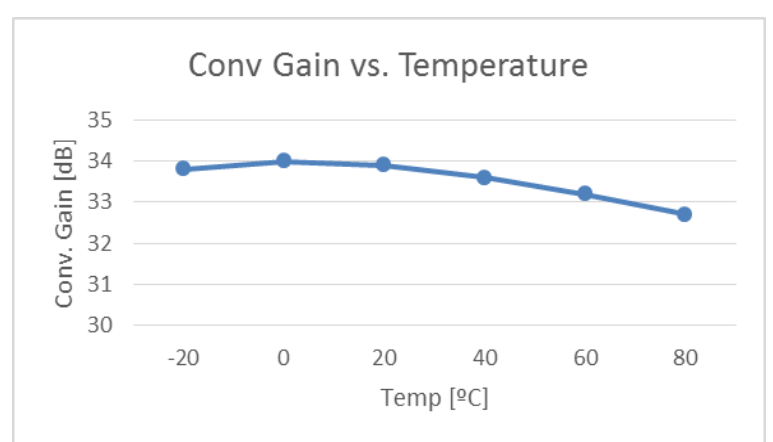

Fig. 10. Measured conversion gain of the X-band LNC vs. temperature.

four Ka-band antenna elements. Signal from each antenna element is fed to one low-noise amplifier (LNA) and these signals are summed on-chip using Wilkinson combiners. The Ka-band mixer down-converts the signal with an off-chip $35.75 \mathrm{GHz} \mathrm{LO}$ signal. In the final stage, the down-converted signal will be filtered using the SMD filters.

The LNAs are designed to minimize the noise figure (NF) which is, with the gain, a critical parameter for the performance of the LNC. Compensation of the bond-wire for the RF signal is done on-chip. Bond-wire inductance is part of the input matching of LNAs for both X- and Ka-band. LNCs for both bands have single-ended RF signal inputs (50 Ohms), single-ended LO input and differential IF output (100 Ohms) to match ADC input impedance. The mixers feature Gilbert cell topology and output buffers feature common collector topology. LNA test chips were fabricated to measure their noise figures and the gain on-wafer. The LNA gains at both bands is another important performance driver as it should be high enough so that the contribution of the noise from the mixer and IF buffer can be negligible. Total noise figure of the

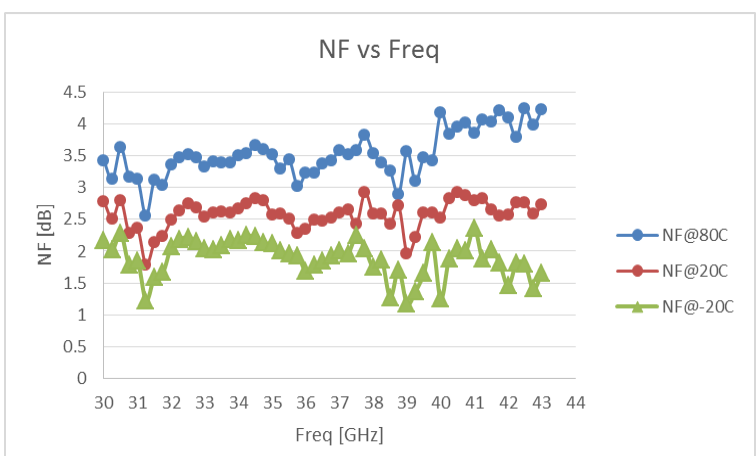

Fig. 11. Measured Ka-band LNA noise figure at $-20,20$ and $80{ }^{\circ} \mathrm{C}$.

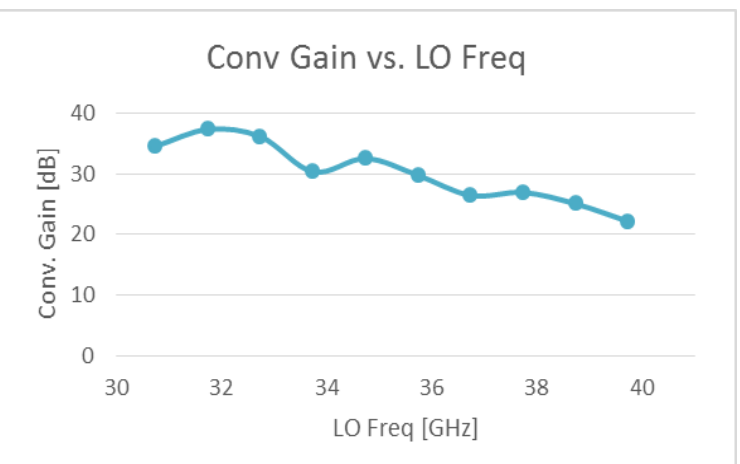

Fig. 12. Measured conversion gain of the Ka-band LNC vs. LO frequency.

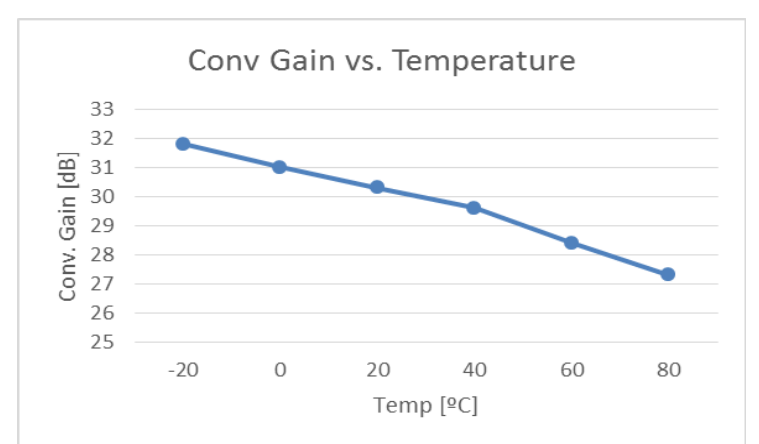

Fig. 13. Measured conversion gain of the Ka-band LNC vs. temperature.

$\mathrm{X}$-band LNC chip is expected to be $3.2 \mathrm{~dB}$, whereas the measured noise figure of the LNA is around $2 \mathrm{~dB}$ at $20^{\circ} \mathrm{C}$. At the Ka-band, the signal-to-noise ratio (SNR) and noise figure can be improved by $3 \mathrm{~dB}$ for each stage of signal summation with Wilkinson combiners. Therefore, the total SNR can be improved by $6 \mathrm{~dB}$.

The X-band LNC draws $25 \mathrm{~mA}$ when biased at $3 \mathrm{~V}$. Simulated NF is $4.8 \mathrm{~dB}$. As it can be observed in Fig. 8, simulated noise figure of the X-band LNA is $1.5 \mathrm{~dB}$ and measured $2.1 \mathrm{~dB}$ at room temperature. Total noise figure of the LNC is estimated to be $5.3 \mathrm{~dB}$. Fig. 9 shows the measured conversion gain of the X-band LNC with different LO frequency. It is observed that a gain of $35.4 \mathrm{~dB}$ is achieved at 9.6 GHz. As shown in Fig. 10, the measured conversion gain of X-band LNC with different temperature. A conversion gain changes by $1.3 \mathrm{~dB}$ from -20 to $80{ }^{\circ} \mathrm{C}$.

Ka-band LNC draws $33 \mathrm{~mA}$ when biased with $3 \mathrm{~V}$. Measured and simulated noise figure of the Ka-band LNA at $35.75 \mathrm{GHz}$ is $2.3 \mathrm{~dB}$ and $2.7 \mathrm{~dB}$ at room temperature, as shown in Fig. 11. The better result shown by the 


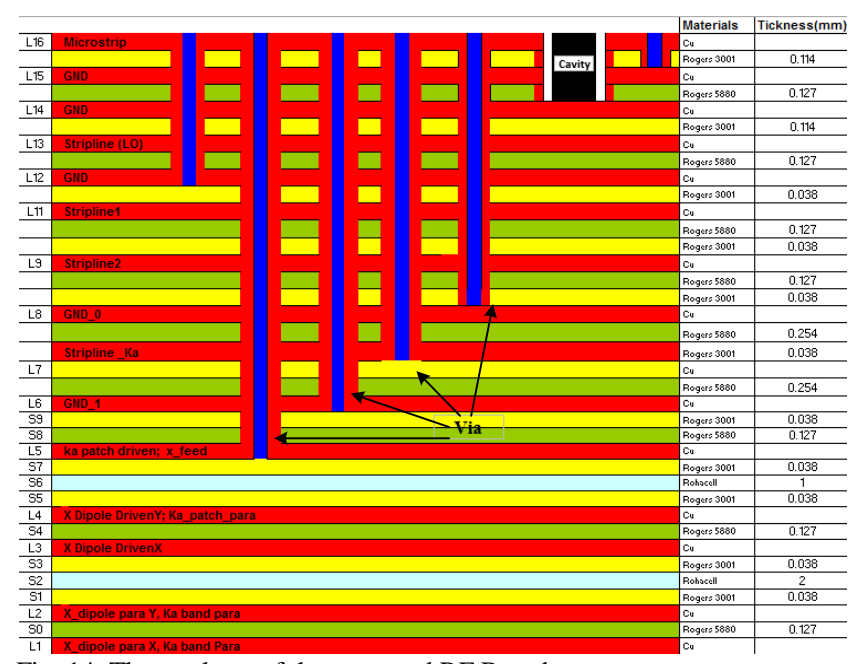

Fig. 14. The stack-up of the proposed RF Board.

measurements is probably due to the ohmic losses which were overestimated in the simulations. Total noise figure of the $\mathrm{Ka}$ band $\mathrm{LNC}$ is estimated to be $0.6 \mathrm{~dB}$. Fig. 12 shows the measured conversion gain of the Ka-band LNC chip. It is observed that $29.7 \mathrm{~dB}$ at $35.75 \mathrm{GHz}$ is achieved. Fig. 13 shows the gain response with different temperature. As can be seen, the conversion gain drops by $4.5 \mathrm{~dB}$ when temperature changes from -20 to $80{ }^{\circ} \mathrm{C}$.

DBFN baseband chip and MMICs are designed and manufactured using IHP's technologies. Due to the demanding frequency range, high performance bipolar transistors are required for MMIC receiver implementation. As a consequence, $130 \mathrm{~nm}$ BiCMOS process has been selected which enables HBTs with $f_{T} / f_{\max }=250 / 300 \mathrm{GHz}$. The DBFN chip have less demanding performance and, for this reason, they have been manufactured using the low-cost $250 \mathrm{~nm}$ BiCMOS technology whose radhard space qualification is currently under investigation.

\section{RF BOARD}

\section{A. Stack-Up Structure}

The RF Board consists of a 16 layers PCB hybrid stack-up where the radiating elements, the MMICs and the distribution networks are integrated. Fig. 14 shows the stack-up configuration of the proposed RF board. The radiating elements are implemented using metal layers from L1 to L7. A laser cavity is realized in the upper part to accommodate MMICs. Each Ka-band patch antenna is fed by the striplines in L7 through the slots in L6. The X-band dipole is fed by microstrip on L5. The striplines and microstrips are connected to the microstrips on L1 via the vertical transition so as to give access the active devices (MMIC).

\section{B. Integrated Feed using X/Ka-Band Dual-Polarized Array \\ 1) X/Ka-band antenna element}

Fig. 15 shows the configuration and the part of stack-up structures (L1 to L8) of the $\mathrm{X}$ - and Ka-band radiating elements. The X-band radiating element is a pair of crossdipole antenna, which is printed on the both sides of a substrate, as shown in Fig. 15(a). The dipoles are proximately

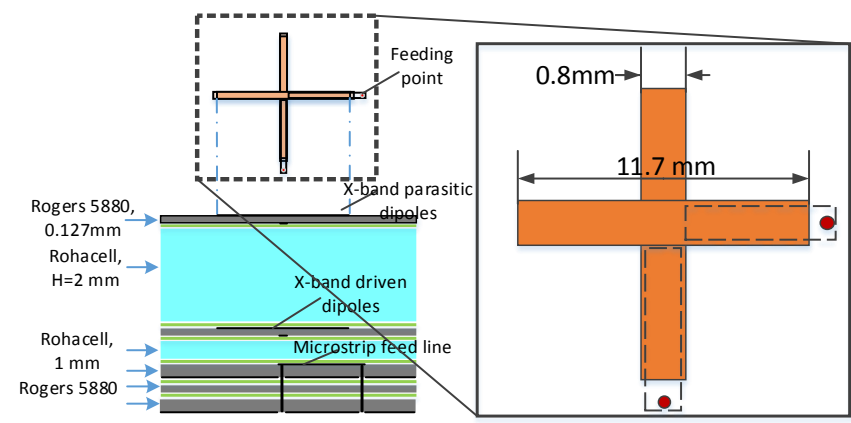

(a)

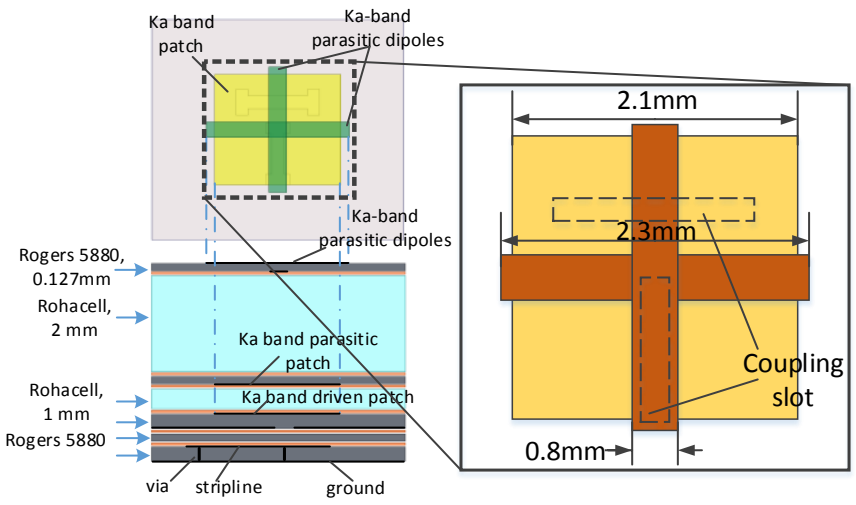

(b)

Fig. 15. The configuration and stack-up of radiating elements: (a) X-band, (b) Ka-band.

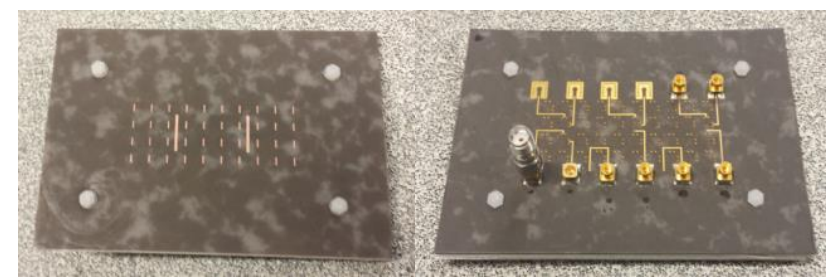

(a)

(b)

Fig. 16. The prototype of the $\mathrm{X} / \mathrm{Ka}$-band dual-polarized subarray: (a) top view, (b) bottom view.

coupled using microstrips. The X-band antenna is designed to work at $9.6 \mathrm{GHz}$ with bandwidth of $300 \mathrm{MHz}$. To further enhance the radiation performance, parasitic dipoles are added above the driven dipoles with a foam of $2 \mathrm{~mm}$ between them. The Ka-band radiating element is a patch antenna, which is fed using stripline through the slots in the ground plane as shown in Fig. 15(b). The patch is designed to work at 35.75 $\mathrm{GHz}$ with the bandwidth over $1 \mathrm{GHz}$. The driven patch of Kaband and the feed of X-band are in the same layer. A pair of cross parasitic dipoles are added on the uppermost board for improving the performance of radiation and gain.

\section{2) $X /$ Ka aperture-shared sub-array}

Based on the X/Ka-band antenna elements design, an X/Kaband dual-polarized sub-array is prototyped and shown in Fig. 16. It is composed of $2 \mathrm{X}$-band element and $4 \times 10 \mathrm{Ka}$-band elements. Fig. 17 shows the simulated and measured Sparameters at X-band and Ka-band respectively. It is observed that the X-band antenna exhibit a good impedance matching performance from 9.3 to $9.9 \mathrm{GHz}$, slightly wider than the simulated ones. The isolation is over $20 \mathrm{~dB}$ between the two 


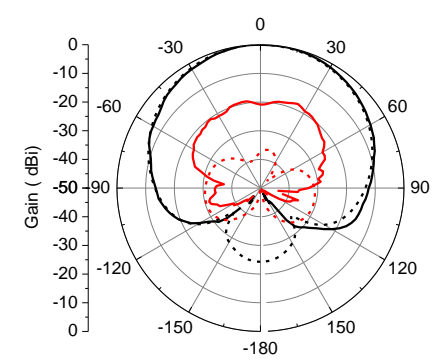

(a)

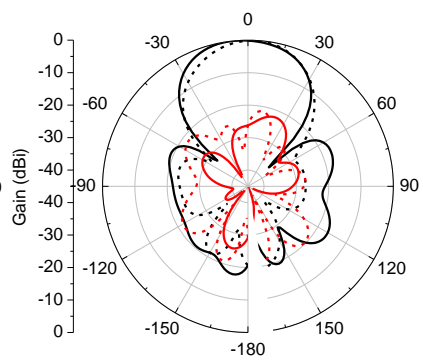

(b)
Fig. 18. The simulated and measured normalized radiation patterns: (a) 9.6 $\mathrm{GHz}$, (b) $35.75 \mathrm{GHz}$.

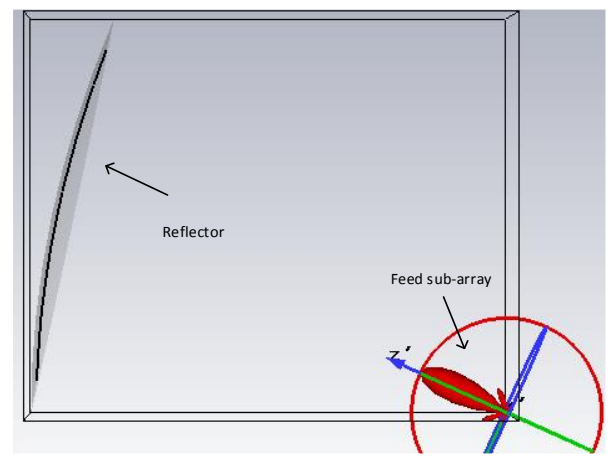

Fig. 19. Configuration of reflector system.

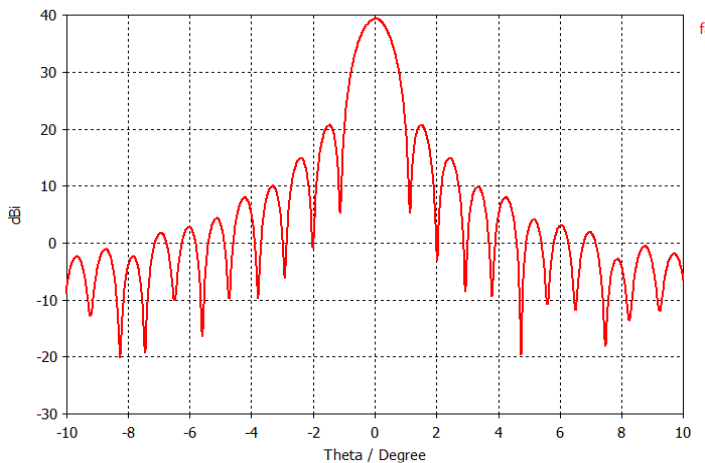

(a)

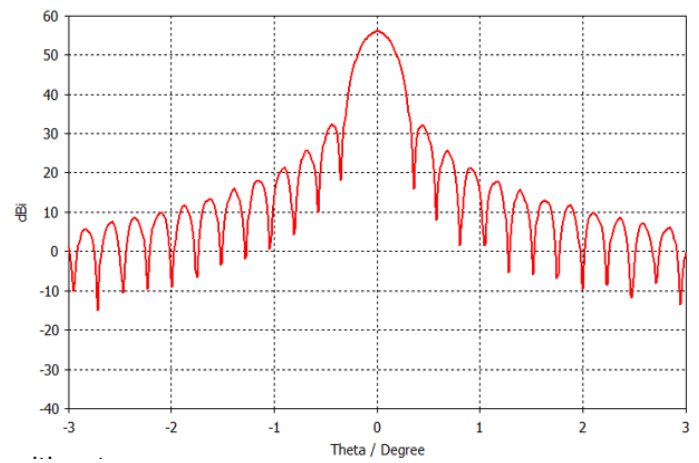

(b)

Fig. 20. The simulated radiation patterns of the reflector (a) $9.6 \mathrm{GHz}$, (b) $35.75 \mathrm{GHz}$.

polarizations. A bandwidth from 34 to $38 \mathrm{GHz}$ is achieved for Ka-band antenna.

Fig. 18 shows the normalized radiation patterns at $9.6 \mathrm{GHz}$ and $35.75 \mathrm{GHz}$, respectively. It is observed excellent radiation performance at $\mathrm{X}$ - and Ka-band is achieved with the cross polarization discrimination (XPD) over $20 \mathrm{~dB}$. It is noted that
X-band channel $(1 \times 1$ element $)$ and Ka-band channel $(2 \times 2$ elements combined) are measured.

\section{3) SAR antenna system including the feed and reflector}

The radiation patterns of the antenna with the reflector included are also investigated. Fig. 19 shows the configuration of the antenna system, which is composed of a paraboloid reflector and a planar feed source. The feed source is the radiation patterns presented in Fig. 18. Fig. 20 presents the radiation patterns of the antenna system at 9.6 and $35.75 \mathrm{GHz}$. It is observed that when the reflector is illuminated with $\mathrm{X}$ band antenna, a gain of $40 \mathrm{dBi}$ and the $3-\mathrm{dB}$ beam width of $0.8^{0}$ are achieved. At the Ka-band operation, the gain is over $56 \mathrm{dBi}$ and the $3-\mathrm{dB}$ beam width is approximately $0.2^{0}$.

\section{CONCLUSION}

In this paper, a novel X/Ka-band dual-polarized DBF-SAR system within the DIFFERENT project is presented. The aim of DIFFERENT is to develop next-generation space-borne SAR systems applied in the future small or micro satellites. The novel SAR concept and techniques such as multi-static, digital beam-forming, reflector-based dual-band dualpolarized aperture-shared antenna array and the integration are presented. Some simulated and measured results of the radiating board, RF frontend, MMIC and digital beamforming network are presented and discussed. The DBF-SAR system has low cost, compact size and high flexibility due to the DBF multi-static SAR architecture and highly integrated RF/digital subsystems, thus it is promising for future SAR missions.

\section{REFERENCES}

[1] W. Imbriale, S. Gao and L. Boccia, Space Antenna Handbook, 2012: Wiley.

[2] P. Zebker, T. Farr, R. Salazar and T. Dixon, "Mapping the world's topography using radar interferometry: the TOPSAR mission," Proc. IEEE, vol. 82, no. 12, pp. 1774-1786, Dec. 1994.

[3] M. Martin, P. Klupar, S. Kilberg and J. Winter, "Techsat 21 and revolutionizing space missions using microsatellites," $15^{\text {th }}$ Am. Inst. Of Aeronaut. And Astronaut Conf. on Small Satellites 2001, Utah, USA, 2001.

[4] D. Massonnet, "Capabilities and limitations of the interferometric cartwheel," IEEE Trans. Geosci. Tomote Sens., vol. 39, no. 3, pp. 506520, Mar. 2001.

[5] N. B. Evatronix IPns, P. Lee and R. Girard, "The RADARSAT-2/3 topographic mission," EUSAR 2002, Cologne, Germany, pp. 37-39, 2002.

[6] W. Imbriale, S. Gao and L. Boccia (eds), Space Antenna Handbook, John Wiley \& Sons, UK, May 2012.

[7] A. Moreira, G. Krieger, M. Younis, I. Hajnsek, K. Papathanassiou, M. Eineder, F. De Zan, "Tandem-L: A mission proposal for monitoring dynamic earth processes", IEEE International Geoscience and Remote Sensing Symposium (IGARSS 2011), pp. 24-29, Jul. 2011.

[8] Grzegorz Adamiuk, Christoph Schaefer, Christian Fischer, Christoph Heer, "SAR Architectures based on DBF for C- and X-band applications", Proceedings of 10th European Conference on Synthetic Aperture Radar (EUSAR 2014), Berlin, Germany, pp. 3-5, Jun. 2014.

[9] Paul A. Rosen, Yunjin Kim, Scott Hensley, Scott Shaffer, Louise Veilleux, James Hoffman, Chung-Lun Chuang, Manab Chakraborty, V.Raju Sagi, R.Satish, Deepak Putrevu, and Rakesh Bhan, "An L- and S-band SAR Mission Concept for Earth Science and Applications", Proceedings of 10th European Conference on Synthetic Aperture Radar (EUSAR 2014), Berlin, Germany, pp. 3-5, Jun. 2014. 
[10] M. Suess, B. Grafmueller, and R. Zahn, "A novel high resolution, wide swath SAR system", IEEE International Geoscience and Remote Sensing Symposium (IGARSS 2001), 2001.

[11] A.Patyuchenko, M. Younis,G. Krieger, Z. Wang, S. Gao, F. Qin, C. Mao, S. Glisic, W. Debski, L. Boccia, G. Amendola, E. Arnieri, M. Krstic and E. Celton, "Highly integrated dual-band digital beamforming synthetic aperture radar," EUMC 2015, Paris, pp. 1-4, Sep. 2015.

[12] S. Gao et all, "Dual-Band Digital Beamforming Synthetic Aperture Radar for Earth Observation," 2015 Asia-Pacific Microw. Conf., accepted, 2015.

[13] M. Süß, B. Grafmüller, and R. Zahn, "A novel high resolution, wide swath SAR," in Proc. IEEE Int. Geoscience and Remote Sensing Symp. IGARSS'01, vol. 3, pp. 1013-1015.

[14] G. Krieger, N. Gebert, and A. Moreira, "Multidimensional waveform encoding: A new digital beamforming technique for synthetic aperture radar remote sensing," IEEE Trans. Geosci. Remote Sensing, vol. 46, no. 1, pp. 31-46, Jan. 2008.

[15] F. Bordoni, M. Younis, E. Varona, N. Gebert, and G. Krieger, "Performance investigation on scan-on-receive and adaptive digital beam-forming for high-resolution wide-swath synthetic aperture radar," in Proc. Int. ITG Workshop SmartAntennas, 2009.

[16] A. Currie and M. Brown, "Wide-swath SAR," Radar and Signal Processing,IEE Proc. F (1988-1993), vol. 139, no. 2, pp. 122-135, 1992.

[17] G. Krieger, N. Gebert, and A. Moreira, "Unambiguous SAR signal reconstruction from non-uniform displaced phase centre sampling," IEEE Geosci. Remote Sensing Lett., vol. 1, no. 4, pp. 260-264, Oct. 2004.

[18] N. Gebert, G. Krieger, and A. Moreira, "Digital beamformingon receive: Techniques and optimization strategies for highresolutionwide-swath SAR imaging," IEEE Trans. Aerosp. Electron.Syst., vol. 45, pp. 564592, 2009.

[19] N. Gebert, F. de Almeida, and G. Krieger, "Airborne demonstrationof multichannel SAR imaging," IEEE Geosci. RemoteSensing Lett., vol. 8, no. 5, pp. 963-967, 2011.

[20] J. Kim, M. Younis, P. Prats-Iraola, M. Gabele, and G. Krieger, "First spaceborne demonstration of digital beamforming for azimuth ambiguity suppression," IEEE Trans. Geosci. Remote Sensing, vol. 51, no. 1, pp. 579-590, Jan. 2013.

[21] G. Krieger, N. Gebert, M. Younis, F. Bordoni, A. Patyuchenko, and A. Moreira, "Advanced concepts for ultra-wide swath SAR imaging," in Proc. European Conf. Synthetic Aperture Radar, Friedrichshafen, Germany, June 2008.

[22] J. T. Kare, "Moving receive beam method and apparatus for synthetic aperture radar," U.S. Patent 6175 326, Jan. 2001

[23] G. Krieger and A. Moreira, "Potential of digital beamforming in bi-and multistatic SAR," in Proc. IEEE Int. Geoscience and Remote Sensing Symp. (IGARSS), 2003, vol. 1, pp. 527-529.

[24] C. Schaefer, C. Heer, and M. Ludwig, "Advanced C-band instrument based on digital beamforming," in Proc. European Conf. Synthetic Aperture Radar (EUSAR), Aachen, Germany, 2010.

[25] J. Li and P. Stoica, MIMO Radar Signal Processing. Hoboken, NJ: Wiley, 2009.

[26] M. Villano, G. Krieger, and A. Moreira, "Staggered-SAR for highresolution wide-swath imaging," in Proc. IET Radar, Glasgow, U.K., 2012.

[27] Heer, C.; Soualle, F.; Zahn, R. \& Reber, R., "Investigations on a new high resolution wide swath SAR concept", Geoscience and Remote Sensing Symposium, 2003. IGARSS '03. Proceedings. 2003 IEEE International, 2003, 1, 521-523 vol.1.

[28] Texas Instruments, "12-BIT, 210 MSPS ADC WITH DDR LVDS/CMOS OUTPUTS”, ADS5527 Datasheet, 2007.

[29] James Tsui: Digital techniques for wideband receivers. SciTech Publishing, 2004.

[30] J. Von Neumann, "Probabilistic Logics", Automata Studies, Princeton University Press, 1956 